A N N A L E S Annales de Bretagne et des Pays de l'Ouest

\title{
Les Rohan, Histoire d'une grande famille
}

\section{Aurélie Chatenet-Calyste}

\section{OpenEdition}

\section{Journals}

Édition électronique

URL : https://journals.openedition.org/abpo/4947

DOI : $10.4000 / a b p o .4947$

ISBN : 978-2-7535-8021-3

ISSN : 2108-6443

\section{Éditeur}

Presses universitaires de Rennes

Édition imprimée

Date de publication : 20 décembre 2019

Pagination : 242-244

ISBN : 978-2-7535-8019-0

ISSN : 0399-0826

\section{Référence électronique}

Aurélie Chatenet-Calyste, «Les Rohan, Histoire d'une grande famille », Annales de Bretagne et des Pays de I'Ouest [En ligne], 126-4 | 2019, mis en ligne le 22 janvier 2020, consulté le 02 janvier 2023. URL : http:// journals.openedition.org/abpo/4947 ; DOI : https://doi.org/10.4000/abpo.4947 
bois des charpentes provient visiblement des émondes même s'il arrive parfois que le foncier autorise le colon à prélever (contre monnaies sonnantes et trébuchantes) quelques pieds d'arbres fonciers sur sa tenue pour édifier tel édifice qui sera précisément indiqué dans la baillée.

L'organisation des bâtiments ancillaires dans l'organisation de la ferme aurait sans doute mérité des développements plus importants. Surtout, au-delà de la présence de granges à ouverture en façade soutenues par des poteaux de bois ou de pierres plus nombreuses dans le Trégor (photos pages 71 à 73) qu'ailleurs en BasseBretagne, il aurait été intéressant de vérifier à partir des déclarations à domaine congéable notamment si les transformations du XIXe siècle avaient aussi concerné les bâtiments d'élevage et de stockage des productions agricoles. À la décharge des auteurs, il faut avouer qu'il est bien difficile de mettre en évidence ces éléments car les bâtiments agricoles les plus modestes, bien qu'ils aient été les plus nombreux, ont rarement été conservés. Le même sort a souvent été réservé aux appentis et remises de facture sommaire qui prenaient appui sur les bâtiments principaux.

L'ouvrage résultant des enquêtes menées par l'Inventaire dans le Trégor présente l'immense mérite d'être accessible tant par le prix proposé (12,90€) que par la facilité de lecture. Malgré son format court, il présente l'essentiel sur les évolutions architecturales des grandes fermes trégorroises. Enfin, on ne peut qu'adhérer à la conclusion des auteurs : ces grandes fermes du Trégor méritent le même statut patrimonial que les chapelles, églises ou manoirs car elles façonnent elles aussi l'identité de la région.

Isabelle GUÉGAN

MEnsion-Rigau, Éric, Les Rohan, Histoire d'une grande famille, Perrin, 2017, 320 p.

" Roi ne puis, prince ne daigne, Rohan suis ", la devise attribuée à cette grande famille bretonne est célèbre et l'historien Éric Mension-Rigau, spécialiste de la noblesse, propose d'en faire l'histoire en mêlant récit historique et analyse sociologique. Cette maison, issue d'une branche cadette des comtes de Porhoët, présente deux caractéristiques principales : une rapide ascension en Bretagne puis en France et une division en deux branches à partir du XvII ${ }^{\mathrm{e}}$ siècle.

L'auteur adopte un plan chronologique qui suit les Rohan du XII ${ }^{\mathrm{e}}$ au $\mathrm{xxI}^{\mathrm{e}}$ siècle en cinq parties et onze chapitres. La première débute avec Alain de Porhoët qui, vers 1120, prend le nom de vicomte de Rohan et fait de la ville éponyme une capitale et une forteresse. Grâce aux alliances nouées avec la famille royale, les Rohan détiennent, au milieu du xve siècle, $1 / 5^{\mathrm{e}}$ de la Bretagne autour de Pontivy et Josselin devenue une place forte de premier plan. Jean II de Rohan, allié à la famille ducale, convoite le trône en intriguant entre le roi de France et le duc de Bretagne. Malgré ce "rêve inaccompli " (p. 77), les Rohan sont puissants, possèdent des charges prestigieuses, un patrimoine vaste et embelli grâce aux travaux menés entre 1490 et 1510 à Josselin. 
Deux tournants rythment l'histoire de la famille au XVI ${ }^{\mathrm{e}}$ siècle : l'extinction de la branche aînée, dont les filles sont mariées aux héritiers des branches cadettes de Gié et Guéméné, et la conversion au protestantisme (chapitre III) d'une partie de la famille, conséquence de leur alliance avec les d'Albret. Les Guéméné, restés catholiques, sont récompensés de leur fidélité à Henri III et leur terre devient une principauté en 1570 avant qu'ils ne prennent le titre de duc de Montbazon en 1588. L'avènement d'Henri IV fait la prospérité des Rohan-Gié. En 1603, la vicomté de Rohan est érigée en duché-pairie et le nouveau duc épouse Marguerite de BéthuneSully, fille unique du ministre. Néanmoins, la reprise des guerres de Religion annonce la disgrâce des ducs de Rohan. En 1629, les biens du duc sont placés sous séquestre et le duc, interdit de séjour en Bretagne, s'exile. Son unique héritière, Marguerite épouse en 1645 Henri de Chabot, un lointain cousin catholique, un cadet issu d'une branche cadette du Poitou. Vue par les Rohan comme une mésalliance et une trahison, l'union est un acte politique voulu par le pouvoir royal afin d'éviter l'avènement d'un nouveau duc protestant. Le XVII ${ }^{\mathrm{e}}$ siècle marque le retour des Rohan dans la sphère catholique et dans les réseaux curiaux (chapitre IV). La famille est alors divisée en deux branches : les Rohan-Chabot et les Rohan-Rohan eux-mêmes subdivisés entre les Rohan-Guéméné, ducs de Montbazon, et les Rohan-Soubise dont la terre est érigée en principauté en 1667 puis en duché-pairie sous le nom de Rohan-Rohan en 1714. À la fin du XVII ${ }^{\mathrm{e}}$ siècle, les Rohan sont influents à la cour grâce à leurs alliances avec les Luynes, les Ventadour, les Colbert et la maison de Lorraine.

Au XVIII ${ }^{\mathrm{e}}$ siècle, des tensions éclatent car les « Rohan-Rohan s'acharnent à écraser les autres branches " (p. 78) et intentent un procès retentissant aux RohanChabot. Au cours du siècle, les écarts se creusent entre les deux branches. Les Rohan-Rohan poursuivent leur ascension en obtenant la qualité de princes étrangers en 1758 et les plus grandes charges à la cour, dans l'armée ou dans l'Église (chapitre V). Ils se succèdent comme princes-évêques de Strasbourg et affirment leur pouvoir dans la pierre avec le palais épiscopal construit par Robert de Cotte entre 1732 et 1742 ou le château de Saverne. La fin du XVIII ${ }^{\mathrm{e}}$ siècle est cependant difficile avec la faillite des Guéméné (1782) et l'affaire du collier (1785). À la Restauration, échouant à récupérer l'héritage du prince de Condé (fils de Charlotte de Rohan), les Rohan-Rohan quittent la France et s'installent en Autriche où leur dernier représentant vit encore aujourd'hui. Les Rohan-Chabot demeurent, eux, "à la marge du pouvoir " (chapitre VI), préférant renforcer leur influence en Bretagne.

Avec la Révolution et la Restauration, les Rohan se partagent en deux tendances politiques : ultraroyaliste pour les Rohan-Chabot; orléaniste pour la branche cadette des comtes de Jarnac. Le second XIX ${ }^{\mathrm{e}}$ siècle annonce leur retour sur un domaine passablement amoindri depuis la Révolution et les difficultés financières (800000 ha en 1789 et 40 ha en 1815). La réinstallation à Josselin (chapitre VIII) et sa restauration à partir des années 1860 atteste du " réancrage des Rohan en Bretagne " (p. 148) où ils se lancent dans la vie politique. Alain de Rohan, député royaliste et catholique du Morbihan pendant 38 ans et maire de Josselin de 1882 à 1914, poursuit les travaux à Josselin. Ici, l'historien retrouve un sujet familier et démontre combien les aménagements néogothiques mettent en scène une " féodalité, idéalisée, terrienne et populaire " (chapitre IX, p. 167). La mort d'Alain de Rohan en 1914 et de son 
fils Josselin en 1916 interrompent cette époque fastueuse et la famille doit faire face " aux défis contemporains " (5e partie) ce qui passe par l'entrée dans le monde du travail et l'ouverture du château au public (chapitre X). C'est aussi une rupture sur le plan politique car il faut attendre les années 1960 pour que les Rohan s'engagent dans le camp gaulliste. Josselin de Rohan devient une figure politique locale indétrônable : il est maire de Josselin (1965-2000), conseiller régional (1982-1998) puis sénateur (1983-2011). L'ouvrage se referme sur un échange avec Josselin de Rohan qui rappelle l'importance " d'assurer la pérennité de Josselin pour maintenir [l'] identité familiale " (p. 283).

$\mathrm{Au}$ final, l'auteur livre un ouvrage riche et foisonnant sur une famille qui a traversé les siècles et les régimes politiques. S'il dresse un portrait minutieux des grandes figures, les limites de l'analyse tiennent à la très large chronologie embrassée et complexifiée par les ramifications de l'arbre généalogique des Rohan où le lecteur peut se perdre, malgré la présence de généalogies. L'on regrettera surtout l'absence de références aux sources alors que le fonds Rohan-Bouillon, par exemple, est vaste et insuffisamment exploité par les historiens. L'étude propose des pistes de recherche suggestives sur les domaines, la gestion et l'aménagement de Josselin au cœur de l'identité des Rohan mais surtout autour des femmes qui jouent un rôle crucial dans la préservation des intérêts familiaux et mériteraient des monographies. Leur pouvoir est polymorphe : religieux car c'est " par les femmes que les Rohan se convertissent à la Réforme " (p. 57), économique avec le rôle de Marguerite de Rohan-Chabot dans la gestion du domaine ou de la duchesse Antoinette " chef d'État-major " de Josselin au xxe siècle (p. 221), politique et militaire avec les figures fortes de Catherine de Parthenay et de Louise de Rohan investie de la charge de Grand Écuyer à la mort de son époux en 1761.

Aurélie CHATENET-CALYSTE

GuIOMAR, Jean-Yves, Le bretonisme. Les historiens bretons au XIXe siècle, Rennes, Presses Universitaires de Rennes, 2019, 457 p. (1e édition : Rennes, Société d'histoire et d'archéologie de Bretagne, 1987, 444 p.)

ForD, Caroline, De la province à la nation. Religion et identité politique en Bretagne, Rennes, Presses Universitaires de Rennes, 2018, 280 p. (traduction de Creating the Nation in Provincial France. Religion and Political Identity in Brittany, Princeton, Princeton University Press, 1993, 255 p.).

Avec la réédition du Bretonisme de Jean-Yves Guiomar, paru pour la première fois en 1987, et la traduction du livre de Caroline Ford, publié sous le titre Creating the Nation in Provincial France en 1993, ce sont deux contributions majeures à l'histoire de la Bretagne et de l'idée nationale qui sont mises à la disposition du public. Le premier était depuis longtemps épuisé et ressurgit sur les étals grâce à un travail d'édition particulièrement soigné. Une remarquable préface de Jean Le Bihan resitue l'ouvrage dans le champ historiographique et dans le parcours intellectuel de l'auteur, un cahier d'illustrations familiarise le lecteur avec les grandes figures 